\title{
Which Offers the Best? Stent Graft or Bare Metal Stent for Endovascular Treatment of Aortoiliac Disease
}

\author{
Ozcan Gur $^{1 *}$, Ozkaramanli Gur Demet ${ }^{2}$, Gurkan Selami1 \\ ${ }^{1}$ Department of Cardiovascular Surgery, Namik Kemal University Medical Faculty, Tekirdag, Turkey \\ ${ }^{2}$ Department of Cardiology, Namik Kemal University Medical Faculty, Tekirdag, Turkey \\ Email: *ozcangur@hotmail.com, dozkarm@yahoo.com,gurkancem@yahoo.com
}

How to cite this paper: Gur, O., Demet, O.G. and Selami, G. (2018) Which Offers the Best? Stent Graft or Bare Metal Stent for Endovascular Treatment of Aortoiliac Disease. World Journal of Cardiovascular Diseases, 8, 489-497.

https://doi.org/10.4236/wjcd.2018.811048

Received: August 17, 2018

Accepted: November 9, 2018

Published: November 12, 2018

Copyright (c) 2018 by authors and Scientific Research Publishing Inc. This work is licensed under the Creative Commons Attribution-NonCommercial International License (CC BY-NC 4.0). http://creativecommons.org/licenses/by-nc/4.0/

\section{Open Access}

\begin{abstract}
Background: In the present study, it was aimed to compare the stent grafts and bare-metal stents in terms of post-procedural patency, clinical recovery and complications in the subjects with symptomatic aorto-iliac arterial disease. Methods: A total of 79 subjects with symptomatic aorto-iliac arterial disease treated with endovascular methods were included in the present study. Forty three subjects received self-expendable bare metal stent (ev3 Protégé stent system, Endovascular Inc., Plymouth, Minnesota, USA) and 36 subjects received PTFE-covered stent graft (Fluency Plus Stent Graft, Bard Peripheral Vascular, Tempe, Arizona). The subjects were compared after and at Months 1, 6, and 12 following the procedure in terms of Rutherford's classification, ankle-arm index (AAI), patency rates, and complications. Results: The subjects receiving bare metal stent and stent graft for aorto-iliac arterial disease were followed for averagely 15 months. For the subjects receiving bare metal stent, primary patency rates at months 1,6 , and 12 were $98 \%, 81 \%$, and $70 \%$, respectively, while secondary patency rate at month 12 was found to be $84 \%$. For the group of stent graft, primary patency rates were found as $97 \%$, $97 \%$, and $92 \%$, respectively and secondary patency rate at month 12 was found to be $94 \%$. Stent grafts were applied at the same time in 2 patients who had metal bare metal because the rupture occurred during the procedure. In the comparison between two groups, the group of stent graft was found to be statistically superior to the other in terms of patency, clinical and post-procedural complications. Conclusion: In conclusion, it was found that the stent grafts were superior to the bare metal stents in terms of patency and complication rates in the subjects with symptomatic aortoiliac disease.
\end{abstract}

\section{Keywords}

Aortoiliac Disease, Stent Graft, Bare Metal Stent, Patency, Complication 


\section{Introduction}

Prevalence of the peripheral arterial disease (PAD) is $4 \%$ in the age of 40 and more and it increases sharply to rise above $14 \%$ after age of 70 [1]. The stenosis or occlusions between the distal aorta and the common femoral artery (CFA) are referred as aorto-iliac arterial disease (AIAD) which is a subgroup of PAD. Treatment of the aorto-iliac disease includes medical, endovascular, and open by-pass surgery (OBS). Although excellent patency rates were achieved by OBS, it has recently been replaced by the endovascular procedures because of its perioperative risk factors and the fact that it is an invasive procedure [2]. For the OBS due to AIAD, mortality rate is $4 \%$ and rate of post-procedural complications is $21 \%$ [3] [4] [5] whereas mortality and morbidity rates have been found to be much lower [6]. Furthermore, the endovascular procedures have become the selected modality for AIAD because of short length of stay in hospital, minimal workforce loss, and acceptable long-term patency rates [7]. The most commonly preferred methods among the endovascular procedures include percutaneous balloon angioplasty (PTA) and stenting (BMS). The studies comparing stenting and PTA in the subjects with AIAD include the meta-analyses reporting that stenting is superior to PTA in terms of long-term patency and low complication rate [8]. In the present study, we aimed at comparing PTFE covered stent grafts and bare metal stents in the subjects with AIAD.

\section{Material and Methods}

In our clinic, 79 aortoiliac artery patients who underwent endovascular methods between 2014-2016 were included in the study following the approval of university ethics committee. 43 cases were treated with self expendable bare metal stent (BMS), (ev3 Protege stent system, Endovascular Inc., Plymouth, Minnesota, United States) and 36 were treated with PTFE coated stent graft (SG), (FLUENCY Plus Stent Graft, Bard Peripheral Vascular, Tempe, Arizona). The demographic and preoperative data of the cases were presented in Table 1.

The inclusion criteria were being above 18 years old, cases with TASC-B, C and D aortoiliac artery lesion, BMS or SG endovascular treatment. The exclusion criteria were life expectancy less than 12 months, TASC A iliac lesion, iliac artery aneurysm, hybrid procedure (the cases who underwent surgery following endovascular treatment), pregnancy, antiaggregant use contraindication. Target occlusive lesions were categorized angiographically as $0 \%-50 \%$ mild, $50 \%-79 \%$ moderate, $80 \%$ - 99\% severe and $100 \%$ occlusion. Following procedure, surgical team evaluated the cases in $1^{\text {st }}, 6^{\text {th }}$, and the $12^{\text {th }}$ months by ankle/arm index, rutherford clinical stage and doppler ultrasonography. During ABI evaluation, tension was measured on bilateral upper extremity, lower extremity dorsalis pedis and posterior tibial artery systolic pressure, the highest pressures were approximated. The cases with lower ABI values and increased symptoms underwent computed tomographical angiography (BTA) or digital substruction angiography (DSA) in the control visit. Narrowing by $60 \%$ and above in the target 
Table 1. Demographic and clinical characteristics.

\begin{tabular}{|c|c|c|c|c|c|c|c|c|c|c|c|c|c|c|c|c|}
\hline & & \multicolumn{7}{|c|}{ BMS } & \multicolumn{7}{|c|}{ SG } & \multirow{3}{*}{$\begin{array}{c}\mathrm{p} \\
0.741\end{array}$} \\
\hline & & \multicolumn{3}{|c|}{ Med. \pm s.s./n-\% } & \multicolumn{4}{|c|}{ Med (Min-Max) } & \multicolumn{3}{|c|}{ Med. \pm s.s./n-\% } & \multicolumn{4}{|c|}{ Med (Min-Mak) } & \\
\hline \multicolumn{2}{|c|}{ Age } & 59.8 & \pm & 8.8 & 61 & 44 & - & 77 & 60.7 & \pm & 7.9 & 60 & 44 & - & 74 & \\
\hline \multirow[b]{2}{*}{ Sex } & Male & 38 & & $88 \%$ & & & & & 32 & & $89 \%$ & & & & & \multirow[b]{2}{*}{0.943} \\
\hline & Female & 5 & & $12 \%$ & & & & & 4 & & $11 \%$ & & & & & \\
\hline $\mathrm{DM}$ & & 22 & & $51 \%$ & & & & & 18 & & $50 \%$ & & & & & 0.918 \\
\hline $\mathrm{HT}$ & & 19 & & $44 \%$ & & & & & 13 & & $36 \%$ & & & & & 0.467 \\
\hline Dyslipidemia & & 25 & & $58 \%$ & & & & & 15 & & $42 \%$ & & & & & 0.145 \\
\hline Smoke & & 33 & & $77 \%$ & & & & & 27 & & $75 \%$ & & & & & 0.857 \\
\hline CAD & & 10 & & $23 \%$ & & & & & 6 & & $17 \%$ & & & & & 0.468 \\
\hline CKD & & 2 & & $5 \%$ & & & & & 3 & & $8 \%$ & & & & & 0.503 \\
\hline CVD & & 1 & & $2 \%$ & & & & & 3 & & $8 \%$ & & & & & 0.225 \\
\hline \multirow{3}{*}{ Symptom } & Cloudication & 21 & & $49 \%$ & & & & & 16 & & $44 \%$ & & & & & 0.697 \\
\hline & Rest pain & 12 & & $28 \%$ & & & & & 17 & & $48 \%$ & & & & & 0.002 \\
\hline & Tissue Lose & 10 & & $23 \%$ & & & & & 3 & & $8 \%$ & & & & & 0.075 \\
\hline \multirow{3}{*}{ TASC } & B & 1 & & $2 \%$ & & & & & 1 & & $3 \%$ & & & & & 0.740 \\
\hline & $\mathrm{C}$ & 19 & & $44 \%$ & & & & & 17 & & $47 \%$ & & & & & \\
\hline & $\mathrm{D}$ & 23 & & $53 \%$ & & & & & 18 & & $50 \%$ & & & & & \\
\hline \multirow[b]{2}{*}{ Site } & Right & 20 & & $47 \%$ & & & & & 26 & & $72 \%$ & & & & & \multirow[b]{2}{*}{0.021} \\
\hline & Left & 23 & & $53 \%$ & & & & & 10 & & $28 \%$ & & & & & \\
\hline
\end{tabular}

Independent sample, $\mathrm{t}$ test/Mann-Whitney u test/Ki-Square test (Fischer test). BMS: Bare Metal Stent, SG: Stent Graft, DM: Diabetes Mellitus, HT: Hypertension, CAD: Coronary artery disease, CKD: Chronic kidney disease, CVD: Cerebrovascular disease.

lesion were considered as restenosis and the intervention was repeated. Primary patency was considered as time from patency lesion to restenosis. Secondary patency was considered as patency time following repeated intervention.

\subsection{Medical Therapy}

All cases undergoing procedure were treated with $300 \mathrm{mg}$ klopidogrel (Plavix, Sanofi, Hoechst, Germany) load followed by daily $300 \mathrm{mg}$ asetilsalisilic acid and $75 \mathrm{mg}$ klopidogrel. When klopidogrel was used before the procedure, loading was not performed.

\subsection{Lesion Access}

Access from iliac artery to target lesion was performed under local anesthesia with the use of shuttle sheath (Cook Medical, Bloomington, Ind) in contralateral femoral artery or brachial artery as antegrad, ipsilateral femoral artery as retrograd. Following sheath replacement, $100 \mathrm{IU} / \mathrm{kg}$ dose bolus heparin was administrated. To confirm BTA findings and evaluate distal bed (femoropopliteal angiography) angiographically before the procedure, peripheral angiography was 
performed. Following the lesional passage by 0.035 or 0.018 hydrophilic guidewire, BMS or SG was replaced. For the decision, the diameters of SG and BMS were measured before the procedure in the lumen BTA of distal side of lesion. Osirix-demo (Pixmeo-Sarl, Bernex, Switzerland) program was used to measure lumen diameters.

\subsection{Statistical Analysis}

Mean, standard deviation, median the lowest, the highest, frequency and percentile values were used for definitive statistics of the data. The distributions of the variables were measured by Kolmogorov Simirnov Test. The quantitative data were analyzed by Mann-Whitney $\mathrm{u}$ test and independent sample $\mathrm{T}$ test. Wilcoxon was used for the analysis of repeated measurements. The qualitative data were analyzed by Chi-square test, Fischer test was used when Chi-square settings could not be established. Kaplan Meier was used for survival analyses. SPSS 22.0 program was used for analyses.

\section{Results}

In our clinic, total of 79 patients who underwent BMS and SG due to symptomatic multi-segment AIAD were included in the study. 43 patients underwent BMS, 36 underwent SG. The patients were followed for approximately 15 months, no significant difference in the follow-up duration was found between two groups (p: 0.252).

No significant difference in terms of age, sex, comorbidity and lesion severity was found between 2 groups; however, the incidence of rupture stent was significantly higher in BMS group as compared to graft group $(\mathrm{p}<0.05)$. Between 2 groups, in terms of clinical complaints, the incidence of resting pain was significantly higher in SG group ( $\mathrm{p}$ 0.05). No significant difference in other symptoms was found. The demographical data were presented in Table 1 comprehensively.

According to TASC classification the cases were classified as follows: in BMS group 42 patients (98\%) as TASC C-D, in SG group 35 patients (97\%) as TASC C-D. No significant difference in lesion severity was found between 2 groups (p: 0.740).

No significant difference between BMS and SG groups by preoperative and $1^{\text {st }}$ month Rutherford classification. However, intra- and inter-SG group significant decreases were observed in 6 and $12^{\text {th }}$ month control visits (p: 0.036, 0.014). In both groups, post-operative values were significantly lower as compared to preoperative Rutherford classification $(\mathrm{p}<0.05)$. When BMS and SG groups were compared by $\mathrm{ABI}$, no significant difference was found preoperative, 1 st. and $6^{\text {th }}$ month, however, there was a significant difference in the $12^{\text {th }}$ month between 2 groups (p: 0.012) (Table 2).

The rates of primary patency period were as follows at 1,6 , and $12^{\text {th }}$ months: in BMS group 98\%, 81\% and 70\%; in SG group 97\%, 97\%, and 92\%, respectively. 
Table 2. Rutherford clinical class, Ankle Brakial Index and patency rate according to the groups.

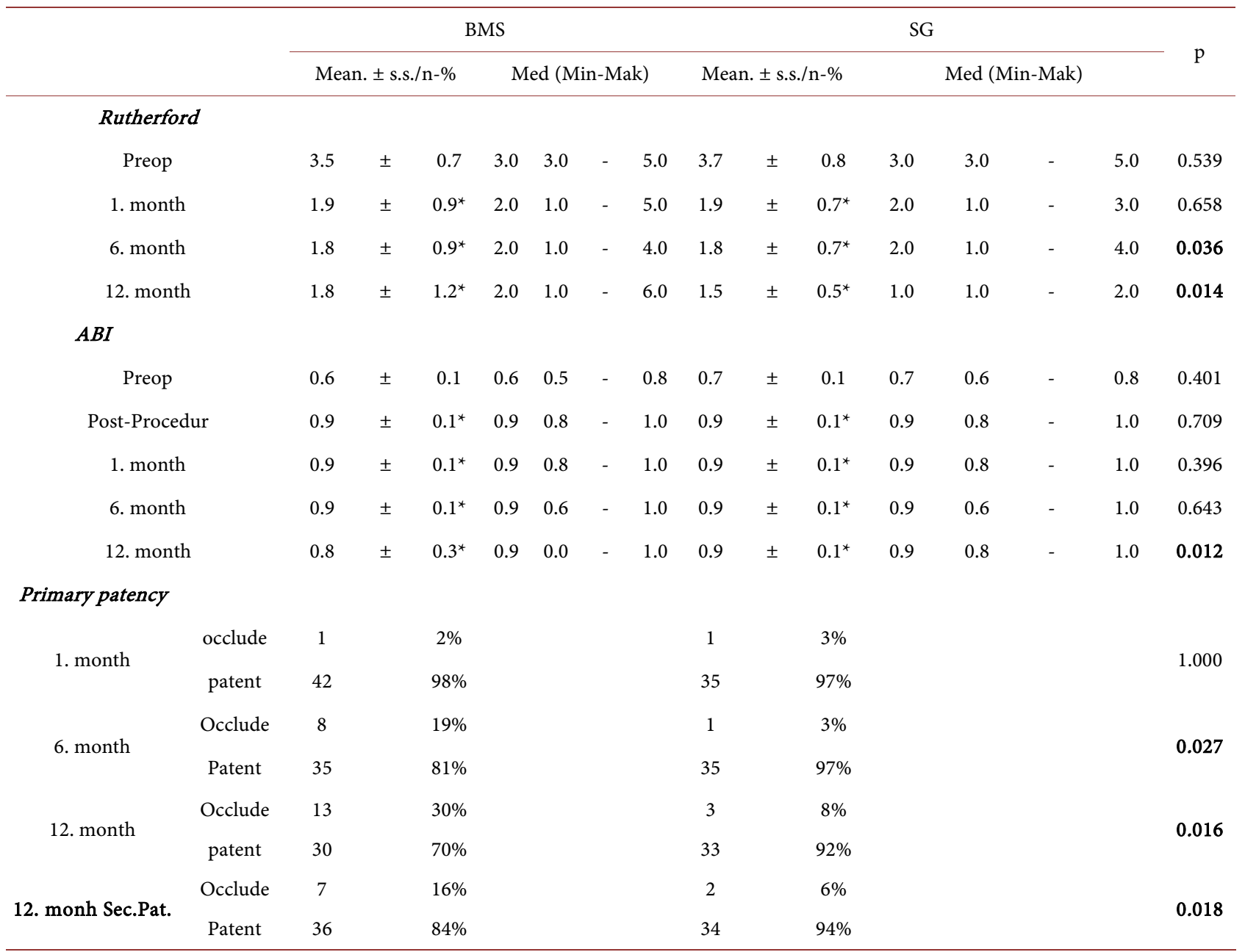

Mann-Whitney u test/Wilcoxon test/Ki-square test (Fischer test); ${ }^{\star} \mathrm{p}<0.05$ statical significantly, BMS: Bare Metal Stent, SG: Stent Graft, Sec. Pat: Secondary Patency.

The rates were significantly higher in SG group at 6 and $12^{\text {th }}$ months. $(\mathrm{p}<0.05)$. In BMS group, 13 (30\%) cases were re-operated, in SG 3 (8\%) cases were reoperated; a significant difference was found between 2 groups (p: 0.002). $12^{\text {th }}$ month secondary patency period was $84 \%$ in BMS group, $\% 94$ in SG group. In terms of secondary patency period, SG was significantly higher (p: 0.018).

When compared in terms of post-operative complications, no mortality was observed in none of the groups. However, in BMS group, a rupture was observed during balloon dilatation in 2 cases without sufficient patency. SG was treated successfully in both cases. Although these 2 patients were accepted as bare metal stent complications, they were not added to the stent graft group during the study and were left out of the study. The complications during the operation were presented in Table 3 comprehensively.

\section{Discussion}

Today, the term "endovascular first" has gained a place in medical sense in the 
Table 3. Procedural features and complication.

\begin{tabular}{|c|c|c|c|c|c|c|c|c|c|}
\hline \multirow[b]{3}{*}{ Dissection } & \multicolumn{4}{|c|}{ BMS } & \multicolumn{4}{|c|}{ SG } & \multirow{3}{*}{$\begin{array}{c}\mathrm{p} \\
0.880\end{array}$} \\
\hline & \multicolumn{2}{|c|}{ Med. \pm s. } & $. / \mathrm{n}-\%$ & \multirow[t]{2}{*}{ Med (Min-Max) } & \multicolumn{3}{|c|}{ Med. \pm s.s. $/ \mathrm{n}-\%$} & \multirow[t]{2}{*}{ Med (Min-Max) } & \\
\hline & 4 & & $9 \%$ & & 3 & & $8 \%$ & & \\
\hline Rupture & 2 & & $5 \%$ & & 0 & & $0 \%$ & 0.880 & \\
\hline Thrombosis & 7 & & $16 \%$ & & 2 & & $5.5 \%$ & 0.028 & 0.135 \\
\hline Mortality & 0 & & $0 \%$ & & 0 & & $0 \%$ & 0.135 & - \\
\hline Amputation & 3 & & $7 \%$ & & 0 & & $0 \%$ & - & 0.246 \\
\hline Reintervention & 13 & & $30 \%$ & & 3 & & $8 \%$ & & 0.002 \\
\hline Stent length & 9.4 & \pm & 3.7 & & 9.2 & \pm & 3.4 & & 0.813 \\
\hline Preop-Drug & 1.4 & \pm & 1.0 & & 1.0 & \pm & 0.9 & & 0.059 \\
\hline $\begin{array}{l}\text { Follow Time } \\
\text { (Month) }\end{array}$ & 14.5 & \pm & 5.2 & & 15.1 & \pm & 4.0 & & 0.252 \\
\hline
\end{tabular}

Independent sample, $\mathrm{t}$ test/Mann-Whitney u test/Ki-Square test (Fischer test); BMS: Bare Metal Stent, SG: Stent Graft, DM: Diabetes Mellitus, HT: Hypertension, CAD: Coronary artery disease, CKD: Chronic kidney disease, CVD: Cerebrovascular disease.

treatment of the cases with AIAD. While the opening of the abdominal cavity reduces morbidity and mortality, the fact that primary and secondary patency rates are comparable with OBS explains why the endovascular procedures should be chosen as the first choice [3] [4] [5]. PTA, BMS and SGI are among the most frequently resorted endovascular methods. 2-year patency rates of PTA for short iliac stenotic segment lesions (TASC-A B) are $80 \%$, while it is around $60 \%$ for 5 year-patency rates and there is not a significant difference detected between PTA and OBS [9] [10]. 325 patients were enrolled in multicenter prospective BRAVISSIMO study which compared PTA and the BMS according to lesion severity levels and while 12 month average primary patency was detected as $93.1 \%$, a significant difference was not detected in the comparison made according to lesion severity level (TASC A-B) between PTA and BMS [11]. Despite the success of BMS and PTA in TASC A-B lesions, the low patency rates in long segments multifocal stenosis, eccentric calsified stenosis and long segment occluded atherosclerotic iliac lesions (TASC C-D) demonstrate that PTA and BMS are not enough in these groups. [12] [13]. While applying BMSs in these advanced AIAD cases, insufficient clarity due to conservative balloon dilatation applications long and problematic vascular bed due to early thrombosis and intimal hyperplasia-related restenosis suggest us that stent grafts may be more successful in this group [14]. In our study, the patency rates in patients who underwent endovascular treatment due to advanced AIAD in $6^{\text {th }}$ and $12^{\text {th }}$ month primary and $12^{\text {th }}$ month secondary follow-ups were seen to be better with SG. In addition, it was also seen that this group of patients had better Rutherford clinical classification and ankle/brachial index results in the clinical follow-up. In a study by Palmaz et al. it was seen that in the cases where SGs used, SGs were seen to keep the calcification away from lumen through the PTFE barrier cov- 
ered in the stent surface and to reduce rate of restenosis by inhibiting the formation of smooth muscle cells dent and intimal hyperplasia [15] [16]. In addition, balloon dilation is applied to the cases with residual stenosis or inadequate stent expansion and intimal damage occurring after this leads to the early thrombosis and intimal hyperplasia as well as restenosis. The fact that intimal damage in SG remains outside the lumen affects restenosis rates positively by reducing the formation of intimal hyperplasia and thrombus. The fact that iliac rupture risk is much lower in SG compared to BMS allows the application of high pressure balloon dilation. This has a positive effect on the long-term patency rates. As for BMS group, there is a rupture risk during balloon dilatation performed due to inadequate stent opening and therefore the required dilatation pressure may not be achieved to enable full opening. Iliac rupture occurred in 2 patients in the BMS group during balloon dilatation performed due to insufficient stent opening, the patients were treated by SG performed to rupture area (Figure 1). The observation that the rates of remaining patent are comparable with surgery in the studies on stent greft use has increased the belief in SGs. Nevelste et al. in a study consisting of 29 patients detected 12-month primary patency rate after aorto-iliac artery disease treatment with SG as $85 \%$, while the secondary patency was detected as 95\%, Cynamon et al. determined the 24 month patency rate as $81 \%$ [14] [17]. In our study, similar results were obtained after the use of SG (12-month primary patency 92\%, secondary patency 94\%). Apart from their outstanding features, the most important criteria that affect their patency rates of SGs are that they are not oversize and that the suffering segment could be closed through antiplatelet treatment. The most common type of failure in SGs is the formation of "edge stenosis". While the most important reason for the formation of this lesion is intimal damage occurring after balloon dilatation, another important one is diameter noncompliance of SG that is used [18]. The

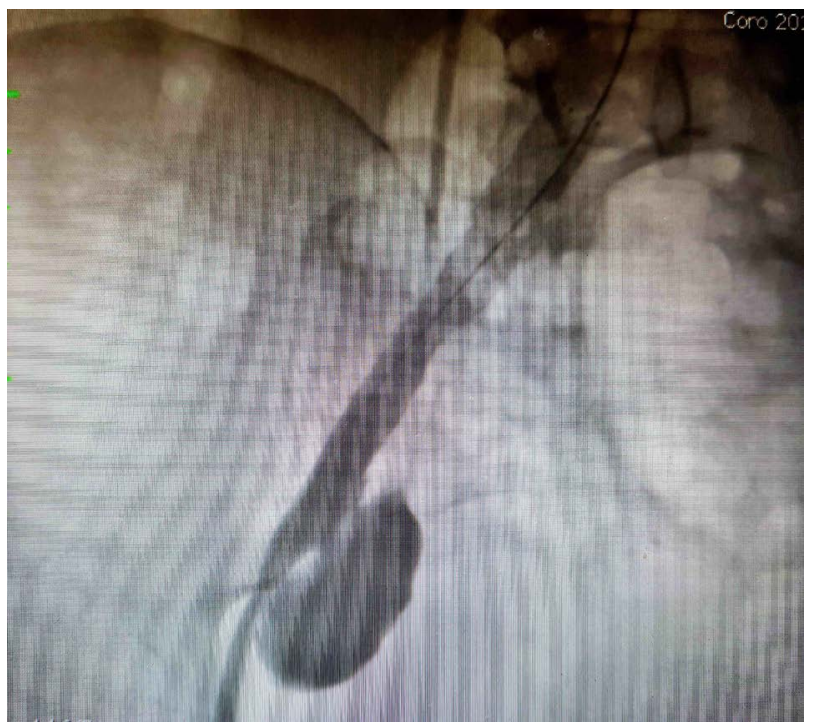

Figure 1. Shows rupture of iliac vein after bare metal stent implantation, which was treated with stent graft. 
diameter of the SG to be applied should be oversize no more than $10 \%$ of the vessel diameter distal to the target lesion. Causing turbulent flow in graft distal, diameter mismatch increases the formation of thrombus and intimal hyperplasia [19]. We used pre-process measurement of CTA of the lesion when determining the diameter of SG to be used in our study. Therefore, we aimed to use the most suitable graft diameter in our study by measuring with CTA taken prior to the procedure. Although the larger delivery system causing larger sheath to be used during the procedure increases access complication risk and this is one of the disadvantages of stent grafts, there was no significant difference in complications related to access region ( $p>0.05$ ). Another disadvantage of SG is that while implementation of BMS in the cases with collateral in their procedure area maintains collateral flow, SGs cause them to be occluded. We are of the opinion that BMS as the primary means will be a better option in the cases where collateral flow must be maintained.

\section{Conclusion}

In the subjects with aorto-iliac arterial disease, BMS and SG achieved successful results with their patency rates similar to the surgery. The fact that rates of mortality and morbidity of the surgery are higher than endovascular inventions leads the endovascular procedures to be preferred in this group of patients. Additionally, the stent grafts were found to be superior to the bare metal stents in terms of higher patency rates and lower complication rates in the subjects with AIAD.

\section{Conflicts of Interest}

There is no conflict of interest and the study has not been published anywhere before.

\section{References}

[1] Selvin, E. and Erlinger, T.P. (2004) Prevalence of and Risk Factors for Peripheral Arterial Disease in the United States: Results from the National Health and Nutrition Examination Survey, 1999-2000. Circulation, 110, 738-743. https://doi.org/10.1161/01.CIR.0000137913.26087.F0

[2] Chiesa, R., Marone, E.M., Tshomba, Y., et al. (2009) Aortobifemoral Bypass Grafting Using Expanded Polytetrafluoroethylene Stretch Grafts in Patients with Occlusive Atherosclerotic Disease. Annals of Vascular Surgery, 23, 764-769. https://doi.org/10.1016/j.avsg.2009.01.004

[3] de Vries, S.O. and Hunink, M.G. (1997) Results of Aortic Bifurcation Grafts for Aortoiliac Occlusive Disease: A Meta-Analysis. Journal of Vascular Surgery, 26, 558-569. https://doi.org/10.1016/S0741-5214(97)70053-3

[4] Passman, M.A., Taylor, L.M., Moneta, G.L., et al. (1996) Comparison of Axillofemoral and Aortofemoral Bypass for Aortoiliac Occlusive Disease. Journal of Vas cular Surgery, 23, 263-269. https://doi.org/10.1016/S0741-5214(96)70270-7

[5] Schneider, J.R., Besso, S.R., Walsh, D.B., et al. (1994) Femorofemoral versus Aortobifemoral Bypass: Outcome and Hemodynamic Results. Journal of Vascular Surgery, 19, 43-55. https://doi.org/10.1016/S0741-5214(94)70119-9 
[6] Dotter, C.T. and Judkins, M.P. (1964) Transluminal Treatment of Arteriosclerotic Obstruction. Description of a New Technic and a Preliminary Report of Its Application. Circulation, 30, 654-670. https://doi.org/10.1161/01.CIR.30.5.654

[7] Palmaz, J.C., Richter, G.M., Noeldge, G., et al. (1988) Intraluminal Stents in Atherosclerotic Iliac Artery Stenosis: Preliminary Report of a Multicenter Study. Radiology, 168, 727-731. https://doi.org/10.1148/radiology.168.3.2970098

[8] Bosch, J.L. and Hunink, M.G. (1997) Meta-Analysis of the Results of Percutaneous Transluminal Angioplasty and Stent Placement for Aortoiliac Occlusive Disease. Radiology, 204, 87-96. https://doi.org/10.1148/radiology.204.1.9205227

[9] Tetteroo, E., van der Graaf, Y., Bosch, J.L., et al. (1998) Randomized Comparison of primary Stent Placement vs Primary Angioplasty Followed by Selective Stent Placement in Patients with Iliac Artery Obstructive Disease. Lancet, 351, 1153-1159. https://doi.org/10.1016/S0140-6736(97)09508-1

[10] Wolf, G.L., Wilson, S.E., Cross, A.P., et al. (1993) Surgery or Balloon Angioplasty for Peripheral Vascular Disease: A Randomized Clinical Trial. Principal Investigators and Their Associates of Veterans Administration Cooperative Study Number 199. Journal of Vascular and Interventional Radiology, 4, 639. https://doi.org/10.1016/S1051-0443(93)71939-9

[11] Bosiers, M., Deloose, K., Callaert, J., et al. (2013) BRAVISSIMO: 12-Month Results from a Large Scale Prospective Trial. Journal of Cardiovascular Surgery (Torino), 54, 235.

[12] Powell, R.J., Fillinger, M., Bettmann, M., et al. (2000) The Durability of Endovascular Treatment of Multisegment Iliac Occlusive Disease. Journal of Vascular Surgery, 31, 1178-1184. https://doi.org/10.1067/mva.2000.104569

[13] Powell, R.J., Fillinger, M., Walsh, D.B., et al. (2000) Predicting Outcomes of Angioplasty and Selective Stenting of Multisegment Iliac Artery Occlusive Disease. Journal of Vascular Surgery, 32, 564-569. https://doi.org/10.1067/mva.2000.107760

[14] Cynamon, J., Marin, M.L., Veith, F.J., et al. (1997) Stent-Graft Repair of Aorto-Iliac Occlusive Disease Coexisting with Common Femoral Artery Disease. Journal of Vascular and Interventional Radiology, 8, 19-26. https://doi.org/10.1016/S1051-0443(97)70508-6

[15] Farb, A., Sangiorgi, G., Carter, A.J., et al. (1999) Pathology of Acute and Chronic Coronary Stenting in Humans. Circulation, 99, 44-52. https://doi.org/10.1161/01.CIR.99.1.44

[16] Palmaz, J.C. (1993) Intravascular Stents: Tissue-Stent Interactions and Design Considerations. American Journal of Roentgenology, 160, 613-618. https://doi.org/10.2214/ajr.160.3.8430566

[17] Nevelsteen, A., Lacroix, H., Stockx, L., et al. (1997) Stent Grafts for Iliofemoral Occlusive Disease. Cardiovascular Surgery, 5, 393-397. https://doi.org/10.1016/S0967-2109(97)00038-0

[18] Endovascular Today Website (2011) Interim 1-Year VIBRANT Results Presented for Gore Viabahn in SFA Treatment. http://bmctoday.net/evtoday/2009/10/article.asp?f=eNews102909_05.htm

[19] Saxon, R.R. (2011) VIPER 1-Year Results in Long SFA Lesions. VIVA 2011: Vascular Interventional Advances (Late-Breaking Clinical Trials), Las Vegas, 18-21 October 2011. 